\title{
An unusual cause of blepharospasm
}

\begin{abstract}
We describe a rare case of multiple dilated Virchow-Robin spaces in the brainstem in a patient presenting initially with blepharospasm with subsequent spread to involve the face and neck. On magnetic resonance imaging (MRI), these lesions demonstrated an isointense signal to cerebrospinal fluid on all sequences with no mass effect or enhancement. Although rare, this condition should be considered part of the differential diagnosis when evaluating cystic abnormalities in the brainstem. This is the first reported case of blepharospasm with subsequent orofacial and neck dystonia caused by dilated Virchow-Robin spaces. The imaging findings and differential diagnoses are discussed.
\end{abstract}

Keyword: Blepharospasm; Virchow-Robin space; Face; Neck 